\title{
Finite Horizon Robustness Analysis of LTV Systems Using Integral Quadratic Constraints
}

\author{
Pete Seiler ${ }^{1} \quad$ Robert M. Moore ${ }^{2} \quad$ Chris Meissen $^{2} \quad$ Murat Arcak $^{3} \quad$ Andrew Packard $^{2}$
}

\begin{abstract}
The goal of this paper is to assess the robustness of an uncertain linear time-varying (LTV) system on a finite time horizon. The uncertain system is modeled as a connection of a known LTV system and a perturbation. The input/output behavior of the perturbation is described by time-domain, integral quadratic constraints (IQCs). Typical notions of robustness, e.g. nominal stability and gain/phase margins, can be insufficient for finite-horizon analysis. Instead, this paper focuses on robust induced gains and bounds on the reachable set of states. Sufficient conditions to compute robust performance bounds are formulated using dissipation inequalities and IQCs. The analysis conditions are provided in two equivalent forms as Riccati differential equations and differential linear matrix inequalities. A computational approach is provided that leverages both forms of the analysis conditions. The approach is demonstrated with two examples.
\end{abstract}

\section{Introduction}

This paper develops theoretical and computational methods to analyze the robustness of linear time-varying (LTV) systems over finite time horizons. Motivating applications for this work include robotic systems [19, 28] and space launch / re-entry vehicles [16, 32] both of which undergo finite-time trajectories. Typical notions of robustness, e.g. nominal stability and gain/phase margins, can be insufficient for such systems. For example, one approach is to evaluate the stability and performance of the LTV system at "frozen" time instances along the trajectory. However, there are LTV systems $\dot{x}(t)=A(t) x(t)$ for which $A(t)$ is stable for each frozen time $t$ but with trajectories that grow unbounded [12].

This paper moves beyond the frozen analysis technique and instead evaluates time-domain metrics over the finite horizon. The analysis is performed on an uncertain LTV

\footnotetext{
${ }^{1} \mathrm{P}$. Seiler is with the Department of AEM at the University of Minnesota, MN, USA. seile017@umn.edu

${ }^{2}$ R. M. Moore, C. Meissen, and A. Packard are with the Department of Mechanical Engineering at the University of California, Berkeley, CA 94720, USA.

\{max.moore, cmeissen, apackard\}@berkeley.edu

${ }^{3}$ M. Arcak is with the Department of Electrical Engineering and Computer Science at the University of California, Berkeley, CA 94720, USA. arcak@berkeley.edu
}

system modeled, as shown in Figure 1, by an interconnection of a known, nominal LTV system $G$ and a perturbation $\Delta$. The perturbation is used to model difficult to analyze elements including nonlinearities and dynamic or parametric uncertainty. The input-output properties of $\Delta$ are characterized by integral quadratic constraints (IQCs) [17]. An extensive list of IQCs for various classes of perturbations is given in $[17,33]$. The main result in [17] is an (infinite-horizon), input-output $\mathcal{L}_{2}$ stability theorem using frequency domain IQCs. The proof relies on an operator theoretic, homotopy method.

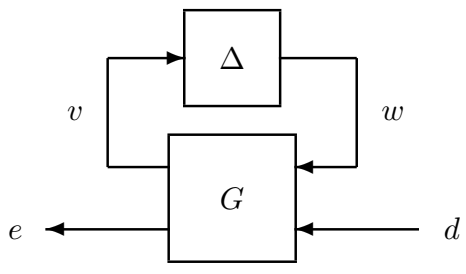

Figure 1: Interconnection $F_{u}(G, \Delta)$ of a nominal LTV system $G$ and perturbation $\Delta$.

The main contribution of this paper is an algorithm to compute robustness metrics on a finite horizon. First, nominal finite-horizon LTV performance is reviewed (Section 2) focusing on induced $\mathcal{L}_{2}$ and $\mathcal{L}_{2}$-to-Euclidean gains. The $\mathcal{L}_{2}$-to-Euclidean gain is useful for computing bounds on the reachable set of states. Next, sufficient conditions are given (Section 3) to bound the induced $\mathcal{L}_{2}$ and $\mathcal{L}_{2}$-to-Euclidean gains for uncertain LTV systems. The analysis is formulated with dissipation inequalities and IQCs. This yields performance conditions in the form of infinite-dimensional differential linear matrix inequalities (DLMIs). These conditions can be equivalently re-written with a Riccati Differential Equation (RDE). This equivalence is based on a variation of the strict bounded real lemma (Theorem 1 in Section 2) which generalizes existing results in $[29,24,10,4]$. The algorithm to compute finite horizon robustness metrics (Section 4) leverages both forms of these conditions. The proposed approach is demonstrated with two examples (Section 5).

This paper adds to existing results to assess robustness of time-varying systems. The most closely related work is [11] which also considers finite horizon robustness with IQCs. However, the work in [11] does not consider disturbances and the theoretical approach / resulting numerical algorithm are different than given here. The work 
in [23] and [9] is also relevant. Uncertain linear parameter varying systems are considered in [23]. The analysis is formulated using dissipation inequalities and IQCs. A similar approach is used in [9] to provide robustness conditions for discrete-time LTV systems. The theoretical and computational approaches provided here differ from these previous works in order to handle continuoustime LTV systems on finite horizons. Other related work includes robustness analysis for finite horizon batch processes [14], nonlinear systems [30], and periodic LTV systems via time-domain lifting $[6,15,13]$ or harmonic balance / frequency domain lifting [34, 7, 27].

Notation: Let $\mathbb{R}^{n \times m}$ and $\mathbb{S}^{n}$ denote the sets of $n$ by- $m$ real matrices and $n$-by- $n$ real, symmetric matrices. The finite-horizon $\mathcal{L}_{2}[0, T]$ norm of a signal $v:[0, T] \rightarrow$ $\mathbb{R}^{n}$ is defined as $\|v\|_{2,[0, T]}:=\left(\int_{0}^{T} v(t)^{T} v(t) d t\right)^{1 / 2}$. If $\|v\|_{2,[0, T]}$ is finite then $v \in \mathcal{L}_{2}[0, T] . \quad \mathbb{R L}_{\infty}$ denotes the set of rational functions with real coefficients that have no poles on the imaginary axis. $\mathbb{R H}_{\infty}$ is the subset of functions in $\mathbb{R L}_{\infty}$ that are analytic in the closed righthalf of the complex plane. Finally, let $E \in \mathbb{S}^{n}$ and $\alpha \in \mathbb{R}$ be given with $E \geq 0$ and $\alpha>0$. Define an ellipsoid in terms of $(E, \alpha)$ as $\mathcal{E}(E, \alpha):=\left\{x \in \mathbb{R}^{n} \mid x^{T} E x \leq \alpha^{2}\right\}$

\section{Nominal Performance}

\subsection{Finite Horizon LTV Systems}

Consider an LTV system $G$ defined on $[0, T]$ :

$$
\begin{aligned}
\dot{x}(t) & =A(t) x(t)+B(t) d(t) \\
e(t) & =C(t) x(t)+D(t) d(t)
\end{aligned}
$$

$x \in \mathbb{R}^{n_{x}}$ is the state, $d \in \mathbb{R}^{n_{d}}$ is the input, and $e \in \mathbb{R}^{n_{e}}$ is the output. The state matrices $A:[0, T] \rightarrow \mathbb{R}^{n_{x} \times n_{x}}, B$ : $[0, T] \rightarrow \mathbb{R}^{n_{x} \times n_{d}}, C:[0, T] \rightarrow \mathbb{R}^{n_{e} \times n_{x}}$, and $D:[0, T] \rightarrow$ $\mathbb{R}^{n_{e} \times n_{d}}$ are piecewise-continuous (bounded) functions of time. It is assumed throughout that $T<\infty$. Thus $d \in$ $\mathcal{L}_{2}[0, T]$ implies $x$ and $e$ are in $\mathcal{L}_{2}[0, T]$ for any $x(0)[3]$.

Many different performance metrics can be defined for this (nominal) finite-horizon LTV system. This paper mainly focuses on two specific metrics. First, the finitehorizon induced $\mathcal{L}_{2}$-gain of $G$ is

$\|G\|_{2,[0, T]}:=\sup \left\{\frac{\|e\|_{2,[0, T]}}{\|d\|_{2,[0, T]}} \mid x(0)=0,0 \neq d \in \mathcal{L}_{2,[0, T]}\right\}$.

As noted above, $d \in \mathcal{L}_{2}[0, T]$ implies $e \in \mathcal{L}_{2}[0, T]$. Thus the $\mathcal{L}_{2}$ gain is finite for any fixed horizon $T<\infty$.

Next, assume $D(T)=0$. Then the finite-horizon $\mathcal{L}_{2}$ to-Euclidean gain of $G$ is

$\|G\|_{E,[0, T]}:=\sup \left\{\frac{\|e(T)\|_{2}}{\|d\|_{2,[0, T]}} \mid x(0)=0,0 \neq d \in \mathcal{L}_{2,[0, T]}\right\}$.

The $\mathcal{L}_{2}$-to-Euclidean gain depends on the system output $e$ only at the final time $T$. The assumption that $D(T)=0$ ensures this gain is well-defined.
The $\mathcal{L}_{2}$-to-Euclidean gain can be used to bound the set of states $x(T)$ reachable by disturbances of a given norm. This reachable set is formally defined as follows:

$$
\mathcal{R}_{\beta}:=\left\{x(T) \mid x(0)=0,\|d\|_{2,[0, T]} \leq \beta\right\} .
$$

If $C(T)=I_{n_{x}}$ and $D(T)=0$ then $e(T)=x(T)$. In this special case, if $\|G\|_{E,[0, T]} \leq \gamma$ then $\|x(T)\|_{2} \leq \gamma\|d\|_{2,[0, T]}$. This implies the reachable set $\mathcal{R}_{\beta}$ is contained in a sphere of radius $\gamma \beta$. More general ellipsoidal bounds on the reachable set can be obtained by proper selection of the output matrices. For example, select $C:=E^{\frac{1}{2}}$ and $D:=$ 0 for some given $E \in \mathbb{S}^{n_{x}}$ with $E \geq 0$. With these choices $\|G\|_{E,[0, T]} \leq \gamma$ implies an ellipsoidal bound on the reachable set: $\mathcal{R}_{\beta} \subseteq \mathcal{E}(E, \beta \gamma)$. The size of the reachable set scales with the norm of the disturbance input. The state $x(t)$ at intermediate times $t \in[0, T]$ can similarly be bounded using the $\mathcal{L}_{2}$-to-Euclidean gain $\|G\|_{E,[0, t]}$.

\subsection{Generic Quadratic Cost}

The two nominal performance metrics introduced above are generalized in Section 3 to assess robustness of uncertain systems. A generic quadratic cost function is defined next in order to unify these various nominal and robust performance metrics. Specifically, let $Q:[0, T] \rightarrow \mathbb{S}^{n_{x}}$, $R:[0, T] \rightarrow \mathbb{S}^{n_{d}}, S:[0, T] \rightarrow \mathbb{R}^{n_{x} \times n_{d}}$, and $F \in \mathbb{R}^{n_{x} \times n_{x}}$ be given. $(Q, S, R)$ are assumed to be piecewise continuous (bounded) functions. A quadratic cost function $J: \mathcal{L}_{2}[0, T] \rightarrow \mathbb{R}$ is defined by $(Q, S, R, F)$ as follows:

$J(d):=x(T)^{T} F x(T)+\int_{0}^{T}\left[\begin{array}{l}x(t) \\ d(t)\end{array}\right]^{T}\left[\begin{array}{rr}Q(t) & S(t) \\ S(t)^{T} & R(t)\end{array}\right]\left[\begin{array}{l}x(t) \\ d(t)\end{array}\right] d t$

subject to: Eq. 1 with $x(0)=0$

The finite-horizon induced $\mathcal{L}_{2}$ gain of $G$ can be related to the quadratic cost $J$ by proper choice of $(Q, S, R, F)$. In particular, let $\gamma>0$ be given and select $Q(t):=$ $C(t)^{T} C(t), S(t):=C(t)^{T} D(t), R(t):=D(t)^{T} D(t)-$ $\gamma^{2} I_{n_{d}}$, and $F:=0$. This yields the following cost function

$$
J(d)=\|e\|_{2,[0, T]}^{2}-\gamma^{2}\|d\|_{2,[0, T]}^{2}
$$

Thus $J(d) \leq 0 \forall d \in \mathcal{L}_{2}[0, T]$ if and only if $\|G\|_{2,[0, T]} \leq \gamma$.

The finite-horizon $\mathcal{L}_{2}$-to-Euclidean gain of $G$ can also be related to the quadratic cost $J$ but with different choices for $(Q, S, R, F)$. Let $\gamma>0$ be given and select $Q(t):=0, S(t):=0, R(t):=-\gamma^{2} I_{n_{d}}$, and $F:=$ $C^{T}(T) C(T)$. This yields the following cost function

$$
J(d)=\|e(T)\|_{2}^{2}-\gamma^{2}\|d\|_{2,[0, T]}^{2}
$$

Thus $J(d) \leq 0 \forall d \in \mathcal{L}_{2}[0, T]$ if and only if $\|G\|_{E,[0, T]} \leq \gamma$.

\subsection{Strict Bounded Real Lemma}

The next theorem states an equivalence between a bound on the quadratic cost $J$ and the existence of a solution 
to a Riccati Differential Equation (RDE) or Riccati Differential Inequality (RDI). The theorem is expressed in terms of strict inequalities and generalizes existing results for the induced $\mathcal{L}_{2}$ gain of LTV systems [29, 24, 10, 4].

Theorem 1. Let $(Q, S, R, F)$ be given with $R(t) \prec 0$ for all $t \in[0, T]$. The following statements are equivalent:

1. $\exists \epsilon>0$ such that $J(d) \leq-\epsilon\|d\|_{2,[0, T]}^{2} \forall d \in \mathcal{L}_{2}[0, T]$.

2. There exists a differentiable function $Y:[0, T] \rightarrow \mathbb{S}^{n}$ such that $Y(T)=F$ and

$\dot{Y}+A^{T} Y+Y A+Q-(Y B+S) R^{-1}(Y B+S)^{T}=0$

This is a Riccati Differential Equation (RDE).

3. There exists $\epsilon>0$ and a differentiable function $P$ : $[0, T] \rightarrow \mathbb{S}^{n}$ such that $P(T) \succeq F$ and

$$
\begin{aligned}
\dot{P} & +A^{T} P+P A+Q \\
& -(P B+S) R^{-1}(P B+S)^{T} \preceq-\epsilon I
\end{aligned}
$$

This is a strict Riccati Differential Inequality (RDI).

Proof. The proof of $(3 \Rightarrow 1)$ is given below as it highlights the dissipation inequality framework. The remainder of the proof is in Appendix A for completeness.

$(\mathbf{3} \Rightarrow \mathbf{1})$ By the Schur complement lemma [2], the RDI and $R(t) \prec 0$ imply $\exists \tilde{\epsilon}>0$ such that

$$
\left[\begin{array}{cc}
\dot{P}+A^{T} P+P A & P B \\
B^{T} P & 0
\end{array}\right]+\left[\begin{array}{cc}
Q & S \\
S^{T} & R
\end{array}\right] \preceq-\tilde{\epsilon} I
$$

Next define a a quadratic storage function $V(x, t):=$ $x^{T} P(t) x$. Let $x(t)$ be a solution of the LTV system (Equation 1) starting from $x(0)=0$ and forced by some input $d \in \mathcal{L}_{2}[0, T]$. Multiply Equation 6 on the left and right by $\left[x(t)^{T} d(t)^{T}\right]$ and its transpose to obtain the following dissipation inequality:

$$
\dot{V}+\left[\begin{array}{l}
x \\
d
\end{array}\right]^{T}\left[\begin{array}{cc}
Q & S \\
S^{T} & R
\end{array}\right]\left[\begin{array}{l}
x \\
d
\end{array}\right] \leq-\tilde{\epsilon}\left[\begin{array}{l}
x \\
d
\end{array}\right]^{T}\left[\begin{array}{l}
x \\
d
\end{array}\right]
$$

Integrate the dissipation inequality from $t=0$ to $t=T$ :

$$
\begin{aligned}
& V(x(T), T)-V(x(0), 0) \\
& +\int_{0}^{T}\left[\begin{array}{l}
x(t) \\
d(t)
\end{array}\right]^{T}\left[\begin{array}{cc}
Q(t) & S(t) \\
S(t)^{T} & R(t)
\end{array}\right]\left[\begin{array}{l}
x(t) \\
d(t)
\end{array}\right] d t \leq-\tilde{\epsilon}\left\|\left[\begin{array}{l}
x \\
d
\end{array}\right]\right\|_{2,[0, T]}^{2}
\end{aligned}
$$

Apply the boundary condition $P(T) \succeq F$ to obtain:

$$
J(d) \leq V(x(0), 0)-\tilde{\epsilon}\|d\|_{2,[0, T]}^{2}
$$

This bound is valid for any $d \in L_{2}[0, T]$. Hence, applying $x(0)=0$ yields $J(d) \leq-\tilde{\epsilon}\|d\|_{2,[0, T]}^{2} \forall d \in \mathcal{L}_{2}[0, T]$.

This theorem assumes zero initial conditions $x(0)=0$. This implies that the initial stored energy $V(x(0), 0)$ is zero and hence is dropped from Equation 8 in the proof.
Non-zero initial conditions can be incorporated by retaining this initial stored energy in the performance bound.

Nominal performance is most easily assessed using the RDE. The performance $J(d) \leq-\epsilon\|d\|_{2,[0, T]}^{2}$ is achieved if the associated RDE exists on $[0, T]$ when integrated backward from $Y(T)=F$. The assumption $R(t) \prec 0$ ensures $R(t)$ is invertible and hence the RDE is well-defined for all $t \in[0, T]$. Thus the solution of the RDE exists on $[0, T]$ unless it grows unbounded. As a concrete example, it was noted in Section 2.2 that $J(d)=\|e\|_{2,[0, T]}^{2}-\gamma^{2}\|d\|_{2,[0, T]}^{2}$ for specific choices of $(Q, S, R, F)$. The matrix $R$, and hence the RDE, depends on the choice of $\gamma$. For a fixed $\gamma>0$, the performance $\|G\|_{2,[0, T]}<\gamma$ is achieved if the associated RDE exists on $[0, T]$ when integrated backward from $Y(T)=0$. The smallest bound on the induced $\mathcal{L}_{2}$ gain can be found via bisection. The RDI will be used later to assess the robustness of uncertain LTV systems.

\section{Robust Performance}

\subsection{Uncertain LTV Systems}

An uncertain, finite-horizon LTV system is given by the interconnection $F_{u}(G, \Delta)$ of a nominal LTV system $G$ and a perturbation $\Delta$ as shown in Figure 1. The LTV system $G$ is described by the following state-space model:

$$
\begin{aligned}
& \dot{x}_{G}(t)=A_{G}(t) x_{G}(t)+B_{G 1}(t) w(t)+B_{G 2}(t) d(t) \\
& v(t)=C_{G 1}(t) x_{G}(t)+D_{G 11}(t) w(t)+D_{G 12}(t) d(t) \\
& e(t)=C_{G 2}(t) x_{G}(t)+D_{G 21}(t) w(t)+D_{G 22}(t) d(t)
\end{aligned}
$$

where $x_{G} \in \mathbb{R}^{n_{G}}$ is the state. The inputs are $w \in \mathbb{R}^{n_{w}}$ and $d \in \mathbb{R}^{n_{d}}$ while $v \in \mathbb{R}^{n_{v}}$ and $e \in \mathbb{R}^{n_{e}}$ are outputs. The state matrices are piecewise continuous (bounded) functions of time with appropriate dimensions, e.g. $A_{G}$ : $[0, T] \rightarrow \mathbb{R}^{n_{G} \times n_{G}}$. The perturbation $\Delta: \mathbf{L}_{2}^{n_{v}}[0, T] \rightarrow$ $\mathbf{L}_{2}^{n_{w}}[0, T]$ is a bounded, causal operator. Well-posedness of the interconnection $F_{u}(G, \Delta)$ is defined as follows.

Definition 2. $F_{u}(G, \Delta)$ is well-posed if for all $x_{G}(0) \in$ $\mathbb{R}^{n_{G}}$ and $d \in \mathbf{L}_{2}^{n_{d}}[0, T]$ there exists unique solutions $x_{G} \in \mathbf{L}_{2}^{n_{G}}[0, T], v \in \mathbf{L}_{2}^{n_{v}}[0, T], e \in \mathbf{L}_{2}^{n_{e}}[0, T]$, and $w \in \mathbf{L}_{2}^{n_{w}}[0, T]$ satisfying Equation (9) and $w=\Delta(v)$ with a causal dependence on $d$.

The perturbation $\Delta$ can have block-structure as is standard in robust control modeling [37]. It can include blocks that are hard nonlinearities (e.g. saturations) and infinite dimensional operators (e.g. time delays) in addition to true system uncertainties. The term "uncertainty" is used for simplicity when referring to $\Delta$.

\subsection{Integral Quadratic Constraints (IQCs)}

IQCs [17] are used to describe the input/output behavior of $\Delta$. They can be formulated in either the frequency or time domain. The time domain formulation 
is more useful for analysis of uncertain time-varying systems. This formulation is based on the graphical interpretation in Figure 2. The inputs and outputs of $\Delta$ are filtered through an LTI system $\Psi$ with zero initial condition $x_{\psi}(0)=0$. The dynamics of $\Psi$ are given as follows:

$$
\begin{aligned}
& \dot{x}_{\psi}(t)=A_{\psi} x_{\psi}(t)+B_{\psi 1} v(t)+B_{\psi 2} w(t) \\
& z(t)=C_{\psi} x_{\psi}(t)+D_{\psi 1} v(t)+D_{\psi 2} w(t)
\end{aligned}
$$

where $x_{\psi} \in \mathbb{R}^{n_{\psi}}$ is the state. A time domain IQC is an inequality enforced on the output $z$ over a finite horizon. The formal definition is given next.

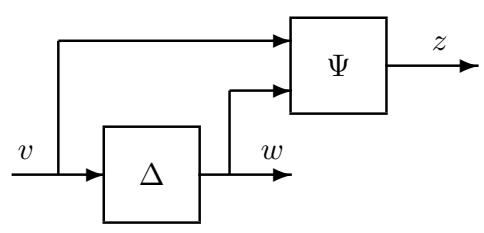

Figure 2: Graphical interpretation for time domain IQCs

Definition 3. Let $\Psi \in \mathbb{R H}_{\infty}^{n_{z} \times\left(n_{v}+n_{w}\right)}$ and $M:[0, T] \rightarrow$ $\mathbb{S}^{n_{z}}$ be given with $M$ piecewise continuous. A bounded, causal operator $\Delta: \mathbf{L}_{2}^{n_{v}}[0, T] \rightarrow \mathbf{L}_{2}^{n_{w}}[0, T]$ satisfies the time domain $I Q C$ defined by $(\Psi, M)$ if the following in-

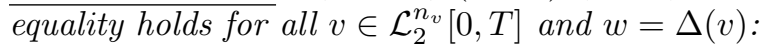

$$
\int_{0}^{T} z(t)^{T} M(t) z(t) d t \geq 0
$$

where $z$ is the output of $\Psi$ driven by inputs $(v, w)$ with zero initial conditions $x_{\psi}(0)=0$.

The notation $\Delta \in \mathcal{I}(\Psi, M)$ is used when $\Delta$ satisfies the corresponding IQC. Time domain IQCs, as defined above, are specified as finite-horizon constraints on the outputs of $\Psi$. These are often referred to as hard IQCs [17]. The definition given here only requires the IQC to hold over the analysis horizon $[0, T]$. This is in contrast to hard IQCs used for infinite horizon analysis which require the constraint to hold over all finite time horizons. Two examples of time domain IQCs are provided below.

Example 4. Consider an LTI uncertainty $\Delta \in \mathbb{R H}_{\infty}$ with $\|\Delta\|_{\infty} \leq 1$. Let $\Pi_{11} \in \mathbb{R} \mathbb{L}_{\infty}$ be given with $\Pi_{11}(j \omega)=$ $\Pi_{11}(j \omega)^{*} \geq 0$ for all $\omega \in \mathbb{R} \cup\{+\infty\}$. Then the following frequency domain IQC holds $\forall v \in L_{2}^{n_{v}}$ and $w=\Delta(v)$

$$
\int_{-\infty}^{\infty}\left[\begin{array}{c}
V(j \omega) \\
W(j \omega)
\end{array}\right]^{*} \underbrace{\left[\begin{array}{cc}
\Pi_{11}(j \omega) & 0 \\
0 & -\Pi_{11}(j \omega)
\end{array}\right]}_{:=\Pi(j \omega)}\left[\begin{array}{c}
V(j \omega) \\
W(j \omega)
\end{array}\right] d \omega \geq 0
$$

where $V$ and $W$ are Fourier transforms of $v$ and $w$. This $I Q C$ corresponds to the use of D-scales used in structured singular value $\mu$ analysis [25, 5, 20, 37]. A factorized representation for $\Pi$ yields a valid time domain
IQC. Specifically, let $\Pi=\Psi^{\sim} M \Psi$ where

$$
\begin{aligned}
\Psi & :=\left[\begin{array}{cc}
\Psi_{11} & 0 \\
0 & \Psi_{11}
\end{array}\right] \text { with } \Psi \in \mathbb{R H}_{\infty}^{n_{z} \times 1} \\
M & :=\left[\begin{array}{cc}
M_{11} & 0 \\
0 & -M_{11}
\end{array}\right] \text { with } M \in \mathbb{S}^{n_{z}} \text { and } M_{11} \succeq 0
\end{aligned}
$$

It is shown in [1] that $(\Psi, M)$ is a valid time domain IQC for $\Delta$ over any finite horizon $T<\infty$.

Example 5. Time domain IQCs are often specified with $\Psi$ as an LTI system and $M$ as a constant matrix. Definition 3 above allows $M$ to be time-varying. This generalization is useful for time-varying real parameters. Let $\Delta:=\delta(t)$ where $\delta(t) \in \mathbb{R}$ and $|\delta(t)| \leq 1$ for all $t \in[0, T]$. Define $\Psi:=I_{2}$ and $M(t):=\left[\begin{array}{cc}m_{11}(t) & 0 \\ 0 & -m_{11}(t)\end{array}\right]$ where $m_{11}:[0, T] \rightarrow \mathbb{R}$ is piecewise continuous and satisfies $m_{11}(t) \geq 0$. Then $\Delta$ satisfies the time domain IQC defined by $(\Psi, M)$. Time-varying IQCs can be defined for other uncertainties, e.g. see related work in [22].

An extensive library of IQCs is provided in [17] for various types of perturbations. Most IQCs are specified in the frequency domain using a multiplier $\Pi$. Under some mild assumptions, a valid time-domain IQC $(\Psi, M)$ can be constructed from $\Pi$ via a $J$-spectral factorization [26]. This allows the library of known (frequency domain) IQCs to be used for time-domain, finite-horizon analysis. More general IQC parameterizations are not necessarily "hard" but can be handled with the method in [8].

\subsection{Robust Induced $\mathcal{L}_{2}$ Gain}

The robustness of $F_{u}(G, \Delta)$ is analyzed using the interconnection shown in Figure 3. The extended system of $G$ (Equation 9) and the IQC filter $\Psi$ (Equation 10) is governed by the following state space model:

$$
\begin{aligned}
\dot{x}(t) & =\mathcal{A}(t) x(t)+\mathcal{B}(t)\left[\begin{array}{l}
w(t) \\
d(t)
\end{array}\right] \\
z(t) & =\mathcal{C}_{1}(t) x(t)+\mathcal{D}_{1}(t)\left[\begin{array}{l}
w(t) \\
d(t)
\end{array}\right] \\
e(t) & =\mathcal{C}_{2}(t) x(t)+\mathcal{D}_{2}(t)\left[\begin{array}{l}
w(t) \\
d(t)
\end{array}\right]
\end{aligned}
$$

The extended state vector is $x:=\left[\begin{array}{l}x_{G} \\ x_{\psi}\end{array}\right] \in \mathbb{R}^{n}$ where $n:=$ $n_{G}+n_{\psi}$. The state-space matrices are given by (dropping the dependence on time $t$ ):

$$
\begin{aligned}
& \mathcal{A}:=\left[\begin{array}{cc}
A_{G} & 0 \\
B_{\psi 1} C_{G 1} & A_{\psi}
\end{array}\right], \mathcal{B}:=\left[\begin{array}{cc}
B_{G 1} & B_{G 2} \\
B_{\psi 1} D_{G 11}+B_{\psi 2} & B_{\psi 1} D_{G 12}
\end{array}\right] \\
& \mathcal{C}_{1}:=\left[\begin{array}{ll}
D_{\psi 1} C_{G 1} & C_{\psi}
\end{array}\right], \mathcal{C}_{2}:=\left[\begin{array}{ll}
C_{G 2} & 0
\end{array}\right], \\
& \mathcal{D}_{1}:=\left[\begin{array}{ll}
D_{\psi 1} D_{G 11}+D_{\psi 2} & D_{\psi 1} D_{G 12}
\end{array}\right] \\
& \mathcal{D}_{2}:=\left[\begin{array}{ll}
D_{G 21} & D_{G 22}
\end{array}\right]
\end{aligned}
$$

The actual system to be analyzed is $F_{u}(G, \Delta)$ with input $d$ and initial condition $x_{G}(0)=x_{G, 0}$. The analysis is instead performed with the extended LTV system (Equation 14) and the constraint $\Delta \in \mathcal{I}(\Psi, M)$. The 


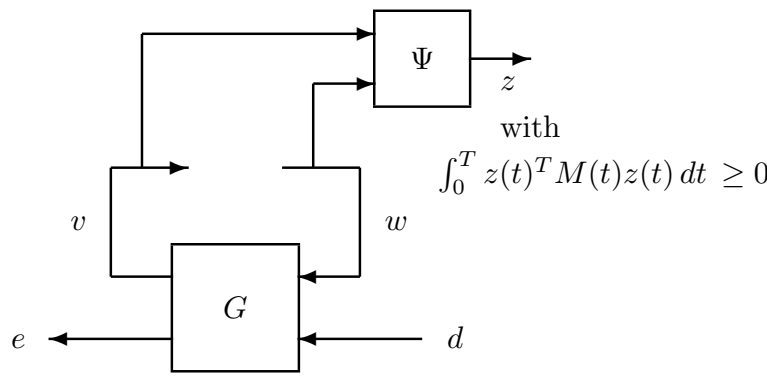

Figure 3: Extended LTI system of $G$ and filter $\Psi$.

constrained extended system has inputs $(d, w)$ and initial condition $x(0)=\left[\begin{array}{c}x_{G, 0} \\ 0\end{array}\right]$. The IQC implicitly constrains the input $w$. The IQC covers $\Delta$ such that the constrained extended system without $\Delta$ includes all behaviors of the original system $F_{u}(G, \Delta)$.

The following differential matrix inequality is used to assess the robust performance of $F_{u}(G, \Delta)^{1}$ :

$$
\begin{gathered}
{\left[\begin{array}{cc}
\dot{P}+\mathcal{A}^{T} P+P \mathcal{A} & P \mathcal{B} \\
\mathcal{B}^{T} P & 0
\end{array}\right]+\left[\begin{array}{cc}
Q & S \\
S^{T} & R
\end{array}\right]} \\
+(\cdot)^{T} M\left[\begin{array}{ll}
\mathcal{C}_{1} & \mathcal{D}_{1}
\end{array}\right] \preceq-\epsilon I
\end{gathered}
$$

This inequality depends on the extended system, IQC, and quadratic cost $(Q, S, R, F)$. It is compactly denoted as $D L M I_{R o b}\left(P, M, \gamma^{2}, t\right) \preceq-\epsilon I$. This notation emphasizes that the constraint is a differential linear matrix inequality (DLMI) in $\left(P, M, \gamma^{2}\right)$ for fixed $(G, \Psi)$ and $(Q, S, R, F)$. The dependence on $(G, \Psi)$ and $(Q, S, R, F)$ is not explicitly denoted but will be clear from context.

The next theorem formulates a sufficient condition to bound the (robust) induced $\mathcal{L}_{2}$ gain of $F_{u}(G, \Delta)$. The proof uses IQCs and a standard dissipation argument $[31,35,36,12]$. For induced $\mathcal{L}_{2}$ gains the quadratic cost matrices are chosen as $F:=0$ and

$$
\begin{aligned}
Q(t) & :=\mathcal{C}_{2}(t)^{T} \mathcal{C}_{2}(t), \quad S(t):=\mathcal{C}_{2}(t)^{T} \mathcal{D}_{2}(t) \\
R(t) & :=\mathcal{D}_{2}(t)^{T} \mathcal{D}_{2}(t)-\gamma^{2}\left[\begin{array}{cc}
0_{n_{w}} & 0 \\
0 & I_{n_{d}}
\end{array}\right]
\end{aligned}
$$

Theorem 6. Let $G$ be an LTV system defined by (9) and $\Delta: \mathcal{L}^{n_{v}}[0, T] \rightarrow \mathcal{L}^{n_{w}}[0, T]$ be a bounded, causal operator. Assume $F_{u}(G, \Delta)$ is well-posed and $\Delta \in \mathcal{I}(\Psi, M)$. If there exists $\epsilon>0, \gamma>0$ and a differentiable function $P:[0, T] \rightarrow \mathbb{S}^{n}$ such that $P(T) \succeq F$ and

$$
D L M I_{R o b}\left(P, M, \gamma^{2}, t\right) \preceq-\epsilon I \quad \forall t \in[0, T]
$$

then $\left\|F_{u}(G, \Delta)\right\|_{2,[0, T]}<\gamma$.

Proof. Let $d \in \mathcal{L}_{2}[0, T]$ and $x_{G}(0)=x_{G, 0}$ be given. By well-posedness, $F_{u}(G, \Delta)$ has a unique solution $\left(x_{G}, v, w, e\right)$. As noted above, the extended system and IQC "cover" this system. In particular, forcing $\Psi$ with

\footnotetext{
${ }^{1}$ The notation $(\cdot)^{T}$ in (15) corresponds to an omitted factor re-
} quired to make the corresponding term symmetric. $(v, w)$ from $x_{\psi}(0)=0$ yields $\left(x_{\psi}, z\right)$. Define $x:=\left[\begin{array}{c}x_{G} \\ x_{\psi}\end{array}\right]$. Then $(x, z, e)$ are a solution of the extended system (14) with inputs $(w, d)$ and initial condition $x(0)=\left[\begin{array}{c}x_{G, 0} \\ 0\end{array}\right]$. Moreover, $z$ satisfies the the IQC defined by $(\Psi, M)$.

Define a storage function by $V(x, t):=x^{T} P(t) x$. Left and right multiply the DLMI (15) by $\left[x^{T}, w^{T}, d^{T}\right]$ and its transpose to show that $V$ satisfies the following dissipation inequality for all $t \in[0, T]$ :

$$
\dot{V}+\left[\begin{array}{c}
x \\
{\left[\begin{array}{l}
w \\
d
\end{array}\right]}
\end{array}\right]^{T}\left[\begin{array}{cc}
Q & S \\
S^{T} & R
\end{array}\right]\left[\begin{array}{c}
x \\
{\left[\begin{array}{l}
w \\
d
\end{array}\right]}
\end{array}\right]+z^{T} M z \leq-\epsilon d^{T} d
$$

Use the choices for $(Q, S, R)$ in (16) to rewrite the second term as $e^{T} e-\gamma^{2} d^{T} d$. Integrate over $[0, T]$ to obtain:

$$
\begin{aligned}
& x(T)^{T} P(T) x(T)-x_{G, 0}^{T} P_{11}(0) x_{G, 0}+\int_{0}^{T} z^{T}(t) M(t) z(t) d t \\
& -\left(\gamma^{2}-\epsilon\right)\|d\|_{2,[0, T]}^{2}+\|e\|_{2,[0, T]}^{2} \leq 0 .
\end{aligned}
$$

Apply $P(T) \succeq F=0$ and $\Delta \in \mathcal{I}(\Psi, M)$ to conclude:

$$
\|e\|_{2,[0, T]}^{2} \leq x_{G, 0}^{T} P_{11}(0) x_{G, 0}+\left(\gamma^{2}-\epsilon\right)\|d\|_{2,[0, T]}^{2}
$$

Finally, if $x_{G}(0)=0$ then $\left\|F_{u}(G, \Delta)\right\|_{2,[0, T]}<\gamma$.

The effect of non-zero initial conditions $x_{G, 0} \neq 0$ is captured in Equation 19. If $d \equiv 0$, this simplifies to $x_{G, 0}^{T} P_{11}(0) x_{G, 0} \geq\|e\|_{2,[0, T]}^{2} \geq 0$ implying $P_{11}(0) \succeq 0$. Furthermore, on $[\tau, T]$ this implies $P_{11}(\tau) \succeq 0$ for all $\tau \in[0, T]$. The IQC is valid only for $x_{\psi}(0)=0$ and hence $P(\tau) \succeq 0$ need not hold in general.

\subsection{Robust $\mathcal{L}_{2}$-to-Euclidean Gain}

A similar theorem provides a bound on the $\mathcal{L}_{2}$-toEuclidean gain of $F_{u}(G, \Delta)$. This requires the additional assumptions that $D_{G 21}(T)=0$ and $D_{G 22}(T)=0$ so that $\mathcal{D}_{2}(T)=0$. Hence $e(T)=\mathcal{C}_{2}(T) x(T)$ and the gain from $d$ to $e(T)$ is well-defined. To assess the robust $\mathcal{L}_{2}$-toEuclidean gain define $(Q, S, R, F)$ as:

$$
\begin{aligned}
& Q(t):=0, S(t):=0, R(t):=-\gamma^{2}\left[\begin{array}{cc}
0_{n_{w}} & 0 \\
0 & I_{n_{d}}
\end{array}\right], \\
& F:=\mathcal{C}_{2}^{T}(T) \mathcal{C}_{2}(T)=\left[\begin{array}{cc}
C_{G 2}(T)^{T} C_{G 2}(T) & 0 \\
0 & 0
\end{array}\right]
\end{aligned}
$$

With these choices for $(Q, S, R, F)$ the next theorem is a minor adaptation of Theorem 6 and the proof is omitted.

Theorem 7. Let $G$ be an LTV system defined by (9) with $D_{G 21}(T)=0$ and $D_{G 22}(T)=0$. Let $\Delta$ : $\mathcal{L}^{n_{v}}[0, T] \rightarrow \mathcal{L}^{n_{w}}[0, T]$ be a bounded, causal operator. Assume $F_{u}(G, \Delta)$ is well-posed and $\Delta \in \mathcal{I}(\Psi, M)$. If there exists $\epsilon>0, \gamma>0$, and a differentiable function $P:[0, T] \rightarrow \mathbb{S}^{n}$ and such that $P(T) \succeq F$ and

$$
D L M I_{R o b}\left(P, M, \gamma^{2}, t\right) \preceq-\epsilon I \quad \forall t \in[0, T]
$$

then $\left\|F_{u}(G, \Delta)\right\|_{E,[0, T]}<\gamma$. 
The condition in Theorem 7 robustly bounds the states $x_{G}(T)$ reachable by disturbances for any uncertainty $\Delta \in$ $\mathcal{I}(\Psi, M)$. Note $\mathcal{C}_{2}(T):=\left[C_{G 2}(T) \quad 0\right]$ so that $e(T)$ only depends on $x_{G}(T)$. The IQC filter $\Psi$ is used only for analysis and $x_{\psi}(T)$ is neglected in the bound.

Robust reachable sets with non-zero initial conditions $x_{G}(0) \neq 0$ can be computed with minor modifications. For example, assume the initial condition of $G$ lies within the ellipsoid $x_{G}(0) \in \mathcal{E}\left(E_{0}, 1\right)$ for some $E_{0} \succ 0$. The IQC still requires $x_{\psi}(0)=0$. Next, enforce $P(0) \preceq$ $\alpha_{1}\left[\begin{array}{rr}E_{0} & 0 \\ 0 & 0\end{array}\right]$ for some $\alpha_{1}>0$ (in addition to the conditions in Theorem 7). It follows from the dissipation inequality proof that the terminal state of $G$ is bounded by $\left\|C_{G 2}(T) x_{G}(T)\right\|_{2}<\alpha_{1}+\gamma\|d\|_{2,[0, T]}$. Additional variations on robust reachable sets with non-zero initial conditions can be found in Chapter 2 of [18].

\subsection{RDE Condition for Robust Performance}

Theorems 6 and 7 provide a DLMI (15) to bound the induced $\mathcal{L}_{2}$ and $\mathcal{L}_{2}$-to-Euclidean gain of $F_{u}(G, \Delta)$. More general robust performance conditions can be formulated by proper choice of $(Q, S, R, F)$. The numerical algorithm proposed in Section 4 relies on an equivalence between the DLMI (15) and a related RDE condition. This equivalence is demonstrated with an extended quadratic cost function $\mathcal{J}$ that combines the performance specification $(Q, S, R, F)$ and the IQC $(\Psi, M)$. Specifically, define $\mathcal{J}$ with the extended dynamics in (14): $\dot{x}=\mathcal{A} x+\mathcal{B}\left[\begin{array}{l}w \\ d\end{array}\right]$. The cost matrices $(\mathcal{Q}, \mathcal{S}, \mathcal{R}, \mathcal{F})$ are chosen as:

$$
\begin{aligned}
& {\left[\begin{array}{cc}
\mathcal{Q} & \mathcal{S} \\
\mathcal{S}^{T} & \mathcal{R}
\end{array}\right]:=(\cdot)^{T} M\left[\begin{array}{ll}
\mathcal{C}_{1} & \mathcal{D}_{1}
\end{array}\right]+\left[\begin{array}{cc}
Q & S \\
S^{T} & R
\end{array}\right]} \\
& \mathcal{F}:=F
\end{aligned}
$$

The quadratic cost associated with these choices is:

$$
\begin{aligned}
\mathcal{J}\left(\left[\begin{array}{l}
w \\
d
\end{array}\right]\right) & :=x(T)^{T} \mathcal{F} x(T)+\int_{0}^{T} z^{T}(t) M(t) z(t) d t \\
& \left.+\int_{0}^{T}\left[\begin{array}{c}
x(t) \\
{\left[\begin{array}{c}
w(t) \\
d(t)
\end{array}\right]}
\end{array}\right]^{T}\left[\begin{array}{cc}
Q(t) & S(t) \\
S(t)^{T} & R(t)
\end{array}\right]\left[\begin{array}{c}
x(t) \\
w(t) \\
d(t)
\end{array}\right]\right] d t
\end{aligned}
$$

The next corollary states the equivalence between the DLMI and RDE conditions. The DLMI can be rewritten as an RDI by the Schur complement lemma [2]. Hence the corollary follows directly from Theorem 1 .

Corollary 8. Let $(\mathcal{Q}, \mathcal{S}, \mathcal{R}, \mathcal{F})$ be given by $(22)$. The following are equivalent for any $\epsilon>0$ and $\gamma>0$ :

1. There exists a differentiable function $P:[0, T] \rightarrow \mathbb{S}^{n}$ such that $P(T) \succeq F$ and $D L M I_{R o b}\left(P, M, \gamma^{2}, t\right) \preceq$ $-\epsilon I$.

2. $\mathcal{R}(t) \prec 0$ for all $t \in[0, T]$. In addition, there exists a differentiable function $Y:[0, T] \rightarrow \mathbb{S}^{n}$ such that $Y(T)=F$ and

$$
\dot{Y}+\mathcal{A}^{T} Y+Y \mathcal{A}+\mathcal{Q}-(Y \mathcal{B}+\mathcal{S}) \mathcal{R}^{-1}(Y \mathcal{B}+\mathcal{S})^{T}=0
$$

\section{Computational Approach}

This section describes computational details and presents an algorithm that combines complementary aspects of the DLMI and RDE robust performance conditions.

\subsection{IQC Parameterization}

There is typically an infinite set of valid IQCs for a given uncertainty $\Delta$. Numerical implementations using IQCs often involve a fixed choice for $\Psi$ and optimization subject to convex constraints on $M[17,33,21]$. The algorithms given in the following sections will use such parameterizations. Two examples are given below.

Example 9. Consider an LTI uncertainty $\Delta \in \mathbb{R H}_{\infty}$ with $\|\Delta\|_{\infty} \leq 1$. By Example 4, $\Delta$ satisfies any $I Q C$ $(\Psi, M)$ with $\Psi:=\left[\begin{array}{cc}\Psi_{11} & 0 \\ 0 & \Psi_{11}\end{array}\right], M:=\left[\begin{array}{cc}M_{11} & 0 \\ 0 & -M_{11}\end{array}\right]$, and $M_{11} \succeq 0$. A typical choice for $\Psi_{11}$ is [33]:

$$
\Psi_{11}^{v}:=\left[1, \frac{1}{(s+p)}, \ldots \frac{1}{(s+p)^{v}}\right]^{T} \text { with } p>0
$$

The robustness analysis is performed by selecting $(p, v)$ to obtain (fixed) $\Psi$ and optimizing over $M_{11} \succeq 0$. The results depend on the choice of $(p, v)$. Larger values of $v$ represent a richer class of IQCs and hence yield less conservative results but with increasing computational cost. Further details on this parameterization are given in [33].

Example 10. The analysis can incorporate conic combinations of multiple IQCs. Let $\left(\Psi_{1}, M_{1}\right)$ and $\left(\Psi_{2}, M_{2}\right)$ define valid IQCs for $\Delta$. Hence $\int_{0}^{T} z_{i}^{T} M_{i} z_{i} d t \geq 0$ where $z_{i}$ is the output $\Psi_{i}$ driven by $v$ and $w=\Delta(v)$. For any $\lambda_{1}, \lambda_{2} \geq 0$ the two constraints can be combined to yield:

$$
\int_{0}^{T} \lambda_{1} z_{1}^{T} M_{1} z_{1}+\lambda_{2} z_{2}^{T} M_{2} z_{2} d t \geq 0
$$

Thus a valid time-domain IQC for $\Delta$ is given by

$$
\Psi:=\left[\begin{array}{l}
\Psi_{1} \\
\Psi_{2}
\end{array}\right] \text { and } M(\lambda):=\left[\begin{array}{cc}
\lambda_{1} M_{1} & 0 \\
0 & \lambda_{2} M_{2}
\end{array}\right]
$$

The analysis is performed by selecting $\left(\Psi_{i}, M_{i}\right)$ and optimizing over $\lambda$.

\subsection{Analysis with the DLMI Condition}

Assume the IQC is $(\Psi, M)$ with $\Psi$ fixed and $M$ constrained to lie within a feasible set $\mathcal{M}$ described by LMIs. The DLMI (15) has the same form for induced $\mathcal{L}_{2}$ and $\mathcal{L}_{2}$-to-Euclidean gains but with different choices of $(Q, S, R, F)$. In both cases the DLMI is linear in $\left(P, M, \gamma^{2}\right)$ for fixed $(G, \Psi)$. The dependence on $\gamma^{2}$ enters via $R$. The best (smallest) bound on the robust gain can be computed from a convex semidefinite program (SDP):

$$
\begin{aligned}
& \min \gamma^{2} \\
& \text { subject to: } M \in \mathcal{M}, P(T) \succeq F \\
& D L M I_{R o b}\left(P, M, \gamma^{2}, t\right) \preceq-\epsilon I \forall t \in[0, T]
\end{aligned}
$$


There are two main issues with solving this SDP. First, the DLMI corresponds to an infinite number of constraints since it must hold for all $t \in[0, T]$. This can be approximated by enforcing the DLMI on a finite time grid $t_{D L M I}:=\left\{t_{k}\right\}_{k=1}^{N_{g}} \subset[0, T]$.

Second, the optimization requires a search over the space of functions $P:[0, T] \rightarrow \mathbb{S}^{n}$. This issue is addressed by restricting $P$ to be a linear combination of differentiable basis functions. Specifically, let $h_{j}:[0, T] \rightarrow \mathbb{R}$ $\left(j=1, \ldots, N_{s}\right)$ and $H_{k}:[0, T] \rightarrow \mathbb{S}^{n}\left(k=1, \ldots, N_{m}\right)$ be given scalar and matrix differentiable basis functions. The storage function and its derivative are given by:

$$
\begin{aligned}
& P(t)=\sum_{j=1}^{N_{s}} h_{j}(t) X_{j}+\sum_{k=1}^{N_{m}} H_{k}(t) x_{k} \\
& \dot{P}(t)=\sum_{j=1}^{N_{s}} \dot{f}_{j}(t) X_{j}+\sum_{k=1}^{N_{m}} \dot{F}_{k}(t) x_{k}
\end{aligned}
$$

Here $\left\{X_{j}\right\}_{j=1}^{N_{s}} \subset \mathbb{S}^{n}$ and $\left\{x_{k}\right\}_{k=1}^{N_{m}} \subset \mathbb{R}$ are optimization variables. Many choices are possible for the basis functions. Initial work in [18] used scalar basis functions generated with a cubic spline and no matrix basis functions. The spline is constructed by selecting an interpolation time grid $\tau_{s p}:=\left\{\tau_{j}\right\}_{j=1}^{N_{s}}$ where $\tau_{j}<\tau_{j+1}$. Note, the spline grid $\tau_{s p}$ is distinct from the DLMI grid $t_{D L M I}$. The spline consists of $N_{s}-1$ cubic functions defined on the intervals $\left[\tau_{j}, \tau_{j+1}\right]$. It interpolates the decision variables $\left\{X_{j}\right\}_{j=1}^{N_{s}}$, i.e. $P\left(\tau_{j}\right)=X_{j}$. The cubic functions satisfy boundary conditions to ensure continuity of the spline and its first/second derivatives at the interval endpoints. The corresponding spline basis functions $\left\{h_{j}\right\}_{j=1}^{N_{s}}$ are not easy to express in explicit form but they can be evaluated numerically at any $t \in[0, T]$. Additional details are given in [18]. The algorithm proposed below also uses a matrix basis function generated by the RDE condition.

The approximations for the DLMI and $P$ lead to a finite dimensional SDP in variables $\left\{X_{j}\right\}_{j=1}^{N_{s}},\left\{x_{k}\right\}_{k=1}^{N_{m}}, M$, and $\gamma^{2}$. The optimization can be performed with standard SDP solvers. Enforcing the DLMI only on a finite grid decreases the optimal cost relative to the original infinite-dimensional SDP. Conversely, restricting $P$ to lie in a finite dimensional subspace increases the optimal cost. The solution accuracy depends on the choice for the constraint time grid and basis functions. A denser time grid and additional bases functions will improve the accuracy but with increased computation time.

\subsection{Analysis with the RDE Condition}

The RDE conditions for (robust) induced $\mathcal{L}_{2}$ and $\mathcal{L}_{2}$-toEuclidean gains do not require the constraint and basis function approximations needed for the corresponding DLMI. Specifically for any $\left(M, \gamma^{2}\right)$ the RDE can be integrated $^{2}$ within a specified numerical accuracy using

\footnotetext{
${ }^{2}$ It is still assumed that $(G, \Psi)$ are given and fixed.
}

standard ODE solvers. If the RDE exists on $[0, T]$ when integrated backward from $Y(T)=F$ then the robust gain is less than $\gamma$. Bisection on $\gamma$ can be used to find the smallest bound on the robust gain. The difficulty with the RDE condition is that IQC matrix $M$ enters in a non-convex fashion. In most cases it would be computationally expensive to perform numerical gradient searches over $M$ to find the smallest bound $\gamma$.

\subsection{Combined Algorithm}

Algorithm 1 combines the DLMI and RDE conditions. The plant $G$ and IQC filter $\Psi$ are given. The algorithm is initialized with a stopping tolerance tol, a max number of iterations $N_{i t e r}$, a time grid $t_{D L M I}$ to enforce the DLMI, a time grid $\tau_{s p}$ for the (scalar) spline basis functions, and a single (zero) matrix basis function $H_{1}$.

The first step is to solve the finite SDP by enforcing the DLMI on $t_{D L M I}$. This returns, if feasible, $\gamma_{S D P}^{(1)}, M^{(1)}$, and the storage function decision variables $\left\{X_{j}^{(1)}\right\}_{j=1}^{N_{s}}$. The next step is to hold the IQC matrix fixed at $M^{(1)}$ and bisect to find the smallest $\gamma$ such that the RDE solution exists on $[0, T]$. This yields $\gamma_{R D E}^{(1)}, P_{R D E}^{(1)}$, and $t_{R D E}^{(1)}$. Here $t_{R D E}^{(1)}$ denotes the (dense) grid of time points returned by the ODE solver associated with $P_{R D E}^{(1)}$.

Two updates are performed before the next iteration. First, the matrix basis function is set equal to the RDE solution if $\gamma_{R D E}^{(1)}<\infty$. This choice is optimal for $M^{(1)}$. At the next iteration the cubic splines are essentially used to perturb around $P_{R D E}^{(1)}$. The second update involves the DLMI time grid. In particular, if the DLMI time grid is too coarse then $\gamma_{S D P}^{(1)}<\gamma_{R D E}^{(1)}$. In this case the DLMI is evaluated on the (dense) grid of time points $t_{R D E}^{(1)}$. The time points where the DLMI is infeasible (or some subset) are added to $t_{D L M I}$. The algorithm terminates if the RDE and SDP results are close or the maximum number of iterations has been reached. Otherwise the subsequent iterations proceed in the same fashion.

\section{Examples}

\subsection{Robust Induced $\mathcal{L}_{2}$ Gain}

Consider an uncertain system $F_{u}(G, \Delta)$ with $\Delta \in \mathbb{R H}_{\infty}$ and $\|\Delta\|_{\infty} \leq 1$. $G$ is an LTI system defined by:

$$
\begin{aligned}
A_{G}:=\left[\begin{array}{cccc}
-0.8 & -1.3 & -2.1 & -2.5 \\
2 & -0.9 & -8.4 & 0.7 \\
2 & 8.6 & -0.5 & 12.5 \\
2 & -0.3 & -12.6 & -0.6
\end{array}\right] & B_{G}:=\left[\begin{array}{cc}
-0.6 & 1 \\
0 & 0.2 \\
0 & 0.4 \\
-1.3 & -0.2
\end{array}\right] \\
C_{G}:=\left[\begin{array}{cccc}
-1.4 & 0 & 0.5 & 0 \\
0 & -0.1 & 1 & 0
\end{array}\right] & D_{G}:=\left[\begin{array}{cc}
-0.3 & 0 \\
0 & 0
\end{array}\right]
\end{aligned}
$$

The infinite-horizon, worst-case induced $\mathcal{L}_{2}$ gain is 1.49 as computed with the wcgain function in Matlab. Finitehorizon robust gains are computed with Algorithm 1. The IQC parameterization in Example 9 is used with $v=1$ and $p=10$. Algorithm 1 is initialized for each 


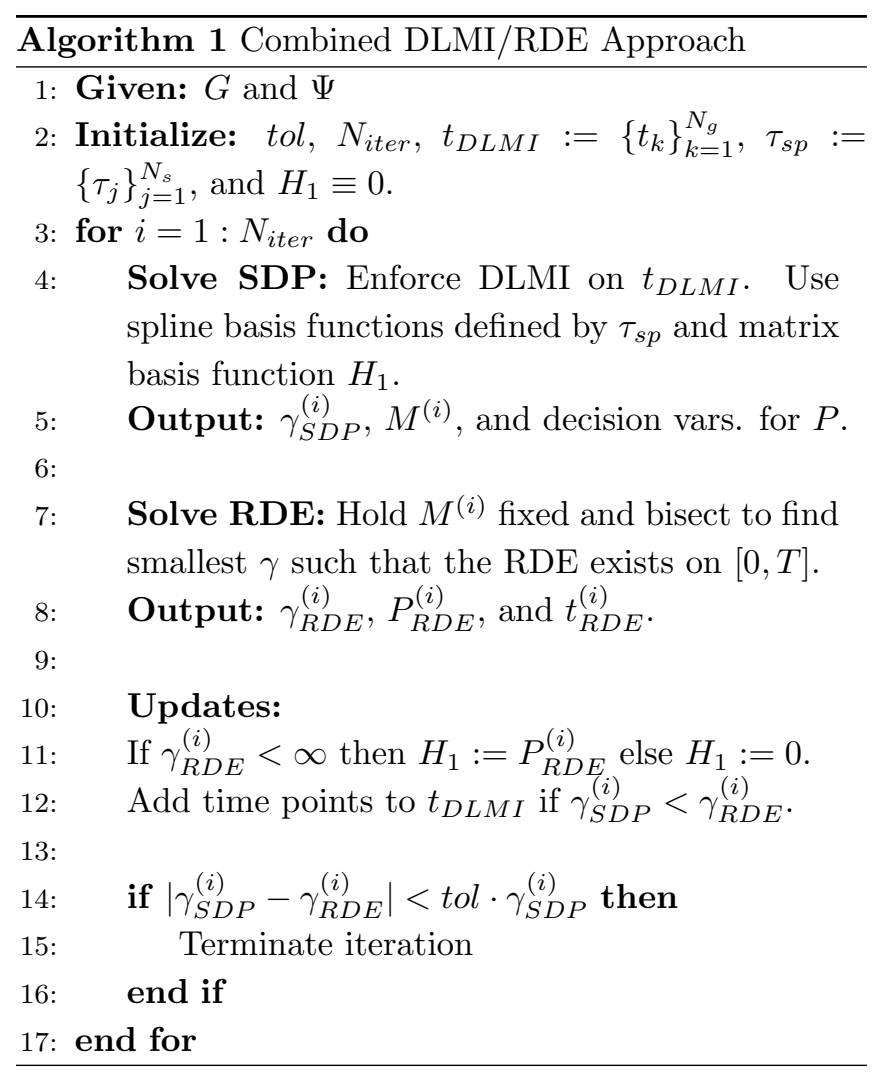

horizon $T$ with tol $=5 \times 10^{-3}, N_{\text {iter }}=10, t_{D L M I}$ as 20 evenly spaced points in $[0, T]$, and $\tau_{s p}$ as 10 evenly spaced points in $[0, T]$. Figure 4 shows the finite-horizon robust gains (blue solid) for $T:=\{1,2,5,10,20,30,40,50,100\}$. The red dashed line denotes the infinite-horizon robust gain of 1.49. It took 466 secs to compute all nine finitehorizon results on a standard laptop. The iteration for $T=5 \mathrm{sec}$ terminated in 3 steps and all other iterations terminated in 2 steps. Matlab's LMILab and ode45 were used to solve the SDP and integrate the RDE in Algorithm 1. The ODE options were set to have an absolute and relative error of $10^{-8}$ and $10^{-5}$, respectively.

\subsection{Two-link robot arm}

This example considers the robustness of a two link robot arm, shown in Figure 5, as it traverses a given finite-time trajectory. The mass and moment of inertia of the $i$-th link are denoted by $m_{i}$ and $I_{i}$. The robot properties are $m_{1}=3 \mathrm{~kg}, m_{2}=2 \mathrm{~kg}, l_{1}=l_{2}=0.3 \mathrm{~m}, r_{1}=r_{2}=0.15 \mathrm{~m}$, $I_{1}=0.09 \mathrm{~kg} \cdot \mathrm{m}^{2}$, and $I_{2}=0.06 \mathrm{~kg} \cdot \mathrm{m}^{2}$. The equations of motion [19] for the two-link robot arm are given by:

$$
\begin{aligned}
& {\left[\begin{array}{cc}
\alpha+2 \beta \cos \left(\theta_{2}\right) & \delta+\beta \cos \left(\theta_{2}\right) \\
\delta+\beta \cos \left(\theta_{2}\right) & \delta
\end{array}\right]\left[\begin{array}{l}
\ddot{\theta}_{1} \\
\ddot{\theta}_{2}
\end{array}\right]+} \\
& {\left[\begin{array}{cc}
-\beta \sin \left(\theta_{2}\right) \dot{\theta}_{2} & -\beta \sin \left(\theta_{2}\right)\left(\dot{\theta}_{1}+\dot{\theta}_{2}\right) \\
\beta \sin \left(\theta_{2}\right) \dot{\theta}_{1} & 0
\end{array}\right]\left[\begin{array}{l}
\dot{\theta}_{1} \\
\dot{\theta}_{2}
\end{array}\right]=\left[\begin{array}{l}
\tau_{1} \\
\tau_{2}
\end{array}\right]}
\end{aligned}
$$

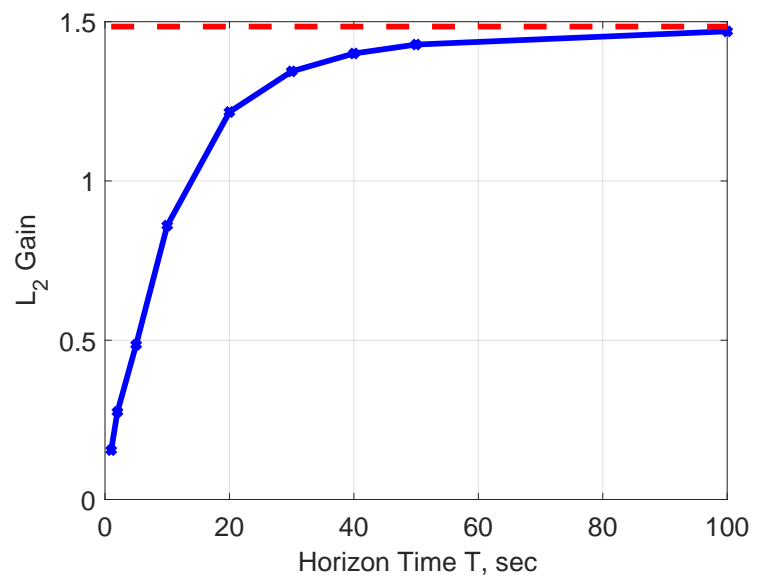

Figure 4: Robust Induced $\mathcal{L}_{2}$ Gain vs. Time Horizon (blue) and infinite horizon result (red dashed)

where $\tau_{i}$ is the torque applied to the base of the $i$-th link and the model parameters are:

$$
\begin{aligned}
\alpha & :=I_{1}+I_{2}+m_{1} r_{1}^{2}+m_{2}\left(l_{1}^{2}+r_{2}^{2}\right)=0.4425 \mathrm{~kg} \cdot \mathrm{m}^{2} \\
\beta & :=m_{2} l_{1} r_{2}=0.09 \mathrm{~kg} \cdot \mathrm{m}^{2} \\
\delta & :=I_{2}+m_{2} r_{2}^{2}=0.105 \mathrm{~kg} \cdot \mathrm{m}^{2}
\end{aligned}
$$

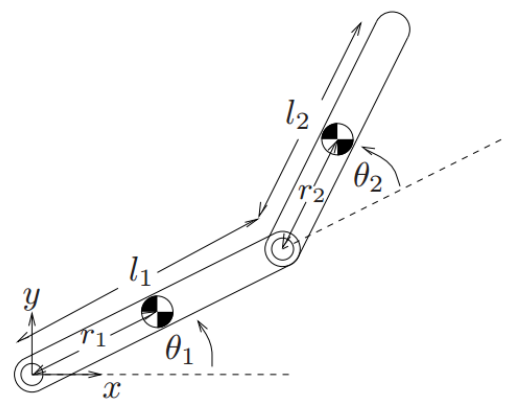

Figure 5: Two link robot arm [19].

The state and input are denoted by $\eta=$ $\left[\begin{array}{llll}\theta_{1} & \dot{\theta}_{1} & \theta_{2} & \dot{\theta}_{2}\end{array}\right]^{T}$ and $\tau=\left[\begin{array}{ll}\tau_{1} & \tau_{2}\end{array}\right]^{T}$. A trajectory $\bar{\eta}$ was selected for the arm and the required input torque $\bar{\tau}$ was computed. Figure 6 shows the desired trajectory for the tip of arm two (red dashed line) in Cartesian coordinates from $t=0$ to $T=5 \mathrm{sec}$. The robot arm positions at four different times are also shown.

The objective is for the robot to track this trajectory in the presence of small torque disturbances $d$. The input torque vector is $\tau=\bar{\tau}+u+d$ where $u$ is an additional control torque (specified below) to reject the disturbances. The nonlinear dynamics (28) are linearized around the trajectory $(\bar{\eta}, \bar{\tau})$ to obtain an LTV system $P$ :

$$
\dot{x}(t)=A(t) x(t)+B(t)(u(t)+d(t))
$$

where $x(t):=\eta(t)-\bar{\eta}(t)$ is the deviation of the nonlinear state from the equilibrium trajectory. The state matrices 


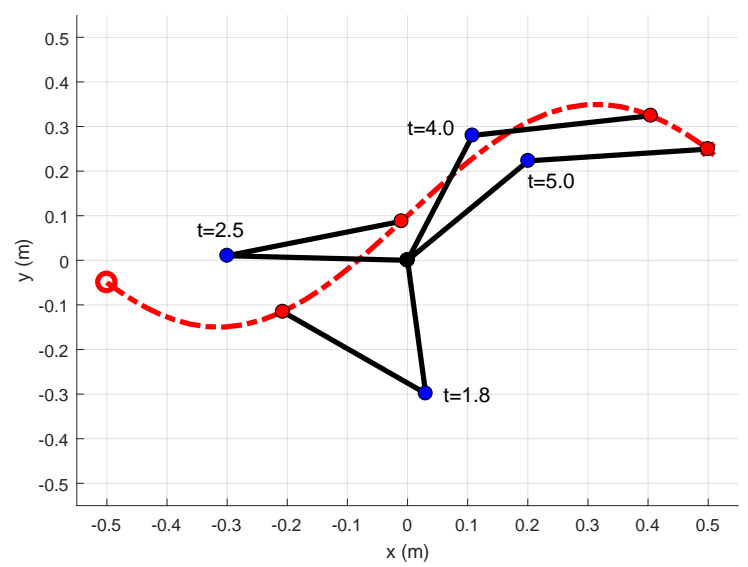

Figure 6: Desired trajectory in Cartesian coordinates (dotted red line) and robot arm position at four times.

$(A, B)$ were computed at 200 uniformly spaced points in $[0,5]$. These state matrices are linearly interpolated to obtain the LTV system at any $t \in[0, T]$.

Next, a time-varying state feedback law $u(t)=$ $-K(t) x(t)$ is designed to improve the disturbance rejection. The feedback gain is constructed via finite horizon, LQR design with the following cost function:

$$
J(x, u)=x(T)^{T} F x(T)+\int_{0}^{T}\left[\begin{array}{l}
x(t) \\
u(t)
\end{array}\right]^{T}\left[\begin{array}{cc}
Q & S \\
S^{T} & R
\end{array}\right]\left[\begin{array}{l}
x(t) \\
u(t)
\end{array}\right] d t
$$

where $Q:=\operatorname{diag}(100,10,100,10), R:=\operatorname{diag}(0.1,0.1)$, $S=0$ and $F:=\operatorname{diag}(1,0.1,1,0.1)$. The optimal feedback gain is $K(t)=R^{-1} B(t)^{T} P(t)$ where $P:[0, T] \rightarrow \mathbb{S}^{n}$ is the solution of the RDE corresponding to $(Q, S, R, F)$ with terminal constraint $P(T)=F$.

The analysis aims to bound the final position of the robot arm in the presence of the disturbances $d$ and uncertainty at the joint connecting the two arms. Figure 7 shows a block diagram for the uncertain, linearized robot arm dynamics. $\Delta \in \mathbb{R H}_{\infty}$ is an LTI uncertainty with $\|\Delta\|_{\infty} \leq 1$. The factors of $\sqrt{0.8}$ are included so that the overall level of uncertainty at the joint is 0.8 . The error signal $e$ contains the two linearized joint angles:

$$
e(t)=\left[\begin{array}{llll}
1 & 0 & 0 & 0 \\
0 & 0 & 1 & 0
\end{array}\right] x(t):=C x(t)
$$

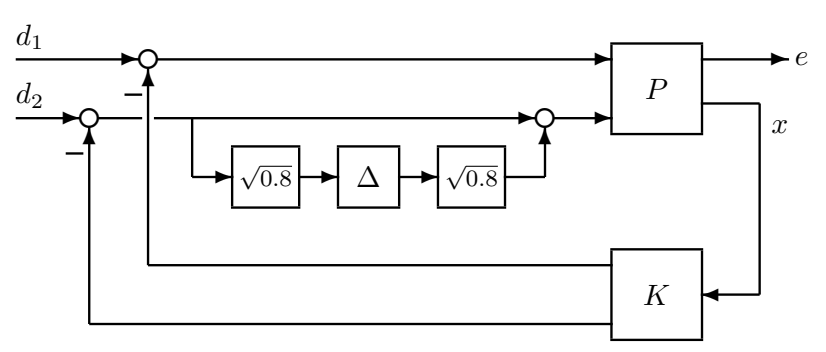

Figure 7: Uncertain LTV Model for Two-Arm Robot

Algorithm 1 was used to compute bounds on the robust $\mathcal{L}_{2}$-to-Euclidean gain from $d$ to $e$ over the $T=5 \mathrm{sec}$ trajectory. The IQC is parameterized as in Example 9 with $v=1$ and $p=10$. Algorithm 1 is initialized with tol $=5 \times 10^{-3}, N_{\text {iter }}=10, t_{D L M I}$ as 20 evenly spaced points in $[0, T]$, and $\tau_{s p}$ as 10 evenly spaced points in $[0, T]$. The algorithm terminated after 3 iterations with a robust gain of $\gamma_{C L}=0.0592$. It took $103 \mathrm{sec}$ to perform this computation. For comparison, the open-loop robust gain (with $K=0$ ) is $\gamma_{O L}=941.6$. This computation terminated in 7 iterations and took 321 sec. As expected, the feedback significantly reduces the gain.

The results were tested by randomly generating 100 instances of $\Delta$ with 0 to 6 states. Each instance of $\Delta$ was substituted into Figure 7 to generate a (nominal) LTV closed-loop. The linearized closed-loop for each $\Delta$ was simulated with disturbances $d$ such that $\|d\|_{2,[0, T]} \leq$ $\beta=5$. Figure 8 shows the linearized simulation results superimposed on the trim trajectory $\bar{\eta}$. The final outputs $e(T)$ are designated by the white dots. The light blue circle corresponds to $\|e(T)\|_{2}^{2}=\theta_{1}(T)^{2}+\theta_{2}(T)^{2} \leq \gamma_{C L}^{2} \beta^{2}$. As expected the simulated trajectories terminate in the computed bound (cyan circle).

Next, the closed-loop gain was evaluated for each $\Delta$ via bisection with the (nominal) RDE. The largest gain was 0.0577 achieved with the following uncertainty:

$$
\Delta_{w c}(s)=\frac{-0.7861 s^{2}-3.383 s-3.631}{0.8 s^{2}+3.414 s+3.631} .
$$

A worst-case disturbance was constructed for the closedloop with $\Delta_{w c}$. This construction is based on the twopoint boundary value problem that connects the performance to the RDE condition (Lemma 11 in Appendix A). This yields a trajectory with terminal condition very near to the boundary of the cyan disk (see zoomed inset) indicating that the computed robustness bounds are not overly conservative. Figure 9 shows the same trajectories and bound but transformed to the Cartesian space of the robot arm.

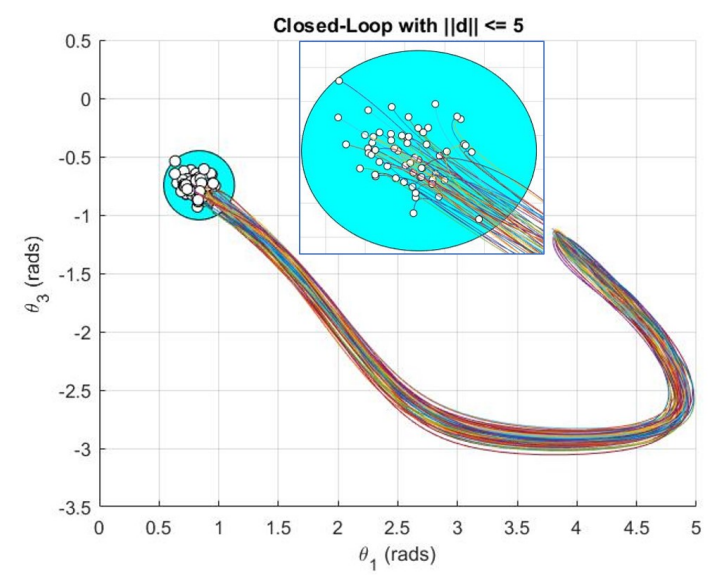

Figure 8: Closed-loop trajectories in the $\left(\theta_{1}, \theta_{2}\right)$ space with $\Delta_{w c}$ and random disturbances $\|d\|_{2,[0, T]} \leq 5$. The robust bound on $e(T)$ is also shown (cyan circle). 


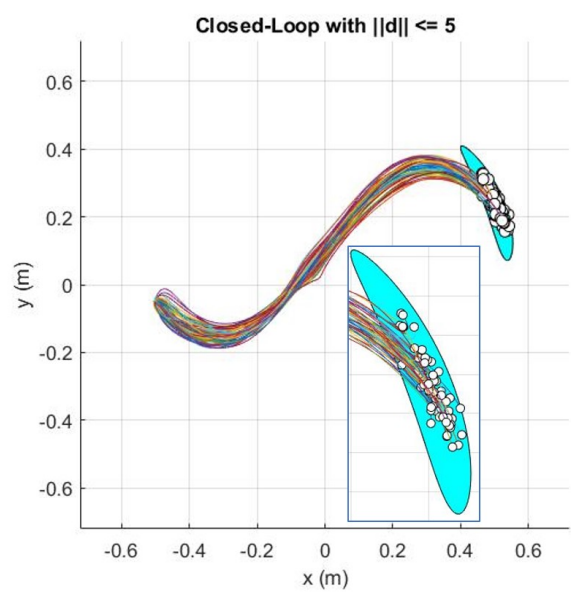

Figure 9: Closed-loop trajectories in Cartesian coordinates with $\Delta_{w c}$ and random disturbances $\|d\|_{2,[0, T]} \leq 5$. The robust bound on $e(T)$ is also shown (cyan circle).

\section{Conclusions}

This paper presented robust performance measures for the analysis of uncertain LTV systems over a finitehorizon. The proposed numerical algorithm combines differential linear matrix inequalities and Riccati differential equations. The utility of robust gains was demonstrated with examples including a two-link robot arm. Future work will include refinements to the algorithm along with methods to construct worst-case perturbations.

\section{Acknowledgments}

The authors gratefully acknowledge support from the National Science Foundation under grants ECCS-1405413 and NSF-CMMI-1254129. A. Packard acknowledges the generous support from the FANUC Corporation.

\section{References}

[1] V. Balakrishnan. Lyapunov functionals in complex $\mu$ analysis. IEEE Transactions on Automatic Control, 47(9):1466-1479, 2002.

[2] S. Boyd, L. E. Ghaoui, E. Feron, and V. Balakrishnan. Linear Matrix Inequalities in System and Control Theory, volume 15 of Studies in Applied Math. SIAM, 1994.

[3] R. Brockett. Finite Dimensional Linear Systems. SIAM Classics in Applied Mathematics, 2015.

[4] W. Chen and F. Tu. The strict bounded real lemma for linear time-varying systems. Journal of Mathematical Analysis and Applications, 244:120-132, 2000.

[5] J. Doyle. Analysis of feedback systems with structured uncertainties. Proc. of IEE, 129-D:242-251, 1982.

[6] G. Dullerud and K. Glover. Robust performance of periodic systems. IEEE Transactions on Automatic Control, 41(8):1146-1159, 1996.
[7] M. Fardad, M. Jovanović, and B. Bamieh. Frequency analysis and norms of distributed spatially periodic systems. IEEE Transactions on Automatic Control, 53(10):2266-2279, 2008.

[8] M. Fetzer, C. Scherer, and J. Veenman. Invariance with dynamic multipliers. submitted to the IEEE Trans. on Automatic Control, 2017.

[9] J. Fry, M. Farhood, and P. Seiler. IQC-based robustness analysis of discrete-time linear time-varying systems. accepted to the International Journal of Robust and Nonlinear Control, 2017.

[10] M. Green and D. J. N. Limebeer. Linear Robust Control. Prentice Hall, 1995.

[11] U. Jönsson. Robustness of trajectories with finite time extent. Automatica, 38:1485-1497, 2002.

[12] H. Khalil. Nonlinear Systems. Prentice Hall, third edition, 2001.

[13] J. Kim, D. Bates, and I. Postlethwaite. Robustness analysis of linear periodic time-varying systems subject to structured uncertainty. Systems and Control Letters, 55(9):719-725, 2006.

[14] D. Ma and R. Braatz. Worst-case analysis of finite-time control policies. IEEE Transactions on Automatic Control, 9(5):766-774, 2001.

[15] L. Ma and P. Iglesias. Quantifying robustness of biochemical network models. BMC Bioinfo., 3(1):38, 2002.

[16] A. Marcos and S. Bennani. LPV modeling, analysis and design in space systems: Rationale, objectives and limitations. In AIAA Guidance, Navigation, and Control Conference, pages AIAA 2009-5633, 2009.

[17] A. Megretski and A. Rantzer. System analysis via integral quadratic constraints. IEEE Transactions on Automatic Control, 42(6):819-830, 1997.

[18] R. Moore. Finite horizon robustness analysis using integral quadratic constraints. Master's thesis, University of California, Berkeley, 2015.

[19] R. Murray, Z. Li, and S. Sastry. A Mathematical Introduction to Robot Manipulation. CRC Press, 1994.

[20] A. Packard and J. Doyle. The complex structured singular value. Automatica, 29:71-109, 1993.

[21] M. Palframan, J. Fry, and M. Farhood. Robustness analysis of flight controllers for fixed-wing unmanned aircraft systems using integral quadratic constraints. submitted to the IEEE Trans. on Control Systems Tech., 2017.

[22] H. Pfifer and P. Seiler. Robustness analysis with parameter-varying integral quadratic constraints. In American Control Conference, pages 138-143, 2015.

[23] H. Pfifer and P. Seiler. Less conservative robustness analysis of linear parameter varying systems using integral quadratic constraints. International Journal of Robust and Nonlinear Control, 26(16):3580-3594, 2016.

[24] R. Ravi, K. Nagpal, and P. Khargonekar. $H_{\infty}$ control of linear time-varying systems: A state-space approach. SIAM J. of Control and Optim., 29(6):1394-1413, 1991. 
[25] M. Safonov. Stability and Robustness of Multivariable Feedback Systems. MIT Press, 1980.

[26] P. Seiler. Stability analysis with dissipation inequalities and integral quadratic constraints. IEEE Transactions on Automatic Control, 60(6):1704-1709, 2015.

[27] S. Shafi, M. Arcak, M. Jovanović, and A. Packard. Synchronization of diffusively-coupled limit cycle oscillators. Automatica, 49:3613-3622, 3013.

[28] M. Spong, S. Hutchinson, and M. Vidyasagar. Robot Modeling and Control. Wiley, 2005.

[29] G. Tadmor. Worst-case design in the time domain: The maximum principle and the standard $H_{\infty}$ problem. Math. of Control, Signals, and Systems, 3:301-324, 1990.

[30] J. Tierno, R. Murray, J. Doyle, and I. Gregory. Numerically efficient robustness analysis of trajectory tracking for nonlinear systems. AIAA Journal of Guidance, Control, and Dynamics, 20(4):640-647, 1997.

[31] A. van der Schaft. $L_{2}$-gain and passivity in nonlinear control. Springer-Verlag New York, Inc., 1999.

[32] J. Veenman, H. Köroğlu, and C. Scherer. Analysis of the controlled NASA HL20 atmospheric re-entry vehicle based on dynamic IQCs. In AIAA Guidance, Navigation, and Control Conference, pages AIAA 2009-5637, 2009.

[33] J. Veenman, C. Scherer, and H. Köroğlu. Robust stability and performance analysis based on integral quadratic constraints. European Journal of Control, 31:1-32, 2016.

[34] N. Wereley and S. Hall. Frequency response of linear time periodic systems. In IEEE Conference on Decision and Control, pages 3650-3655, 1990.

[35] J. Willems. Dissipative dynamical systems part I: General theory. Archive for Rational Mech. and Analysis, 45(5):321-351, 1972.

[36] J. Willems. Dissipative dynamical systems part II: Linear systems with quadratic supply rates. Archive for Rational Mech. and Analysis, 45(5):352-393, 1972.

[37] K. Zhou, J. C. Doyle, and K. Glover. Robust and Optimal Control. Prentice-Hall, 1996.

\section{A Proof of Theorem 1}

Theorem 1 states an equivalence between: 1) a bound on the quadratic cost $J, 2$ ) the existence of a solution $Y$ to a RDE, and 3) the existence of a solution $P$ to a RDI. The proof of $(3 \Rightarrow 1)$ is given in the main text and the rest of the proof is in this appendix. Section A.1 discusses a related two-point boundary value problem (TPBVP). The remainder of the appendix provides proofs for $(1 \Rightarrow$ $2),(2 \Rightarrow 1)$, and $(1 \Rightarrow 3)$. This demonstrates $(1 \Leftrightarrow 2)$ and $(1 \Leftrightarrow 3)$. The equivalence $(2 \Leftrightarrow 3)$ follows from these results. The TPBVP lemma and proof of $(1 \Rightarrow 2)$ is similar to the presentation given in Section 3.7.4 of [10] for the special case of finite-horizon induced $\mathcal{L}_{2}$ gains.

\section{A.1 Two-Point Boundary Value Problem}

The LTV dynamics (Equation 1) and quadratic cost $J$ are defined by $(A, B)$ and $(Q, S, R, F)$, respectively. Define a time-varying Hamiltonian $H:[0, T] \rightarrow \mathbb{S}^{2 n_{x}}$ as:

$$
H:=\left[\begin{array}{cc}
A & 0 \\
-Q & -A^{T}
\end{array}\right]+\left[\begin{array}{c}
-B \\
S
\end{array}\right] R^{-1}\left[\begin{array}{ll}
S^{T} & B^{T}
\end{array}\right]
$$

A two-point boundary value problem (TPBVP) is defined for $t_{0} \in[0, T]$ as:

$$
\begin{aligned}
{\left[\begin{array}{c}
\dot{x}^{*}(t) \\
\dot{\lambda}(t)
\end{array}\right] } & =H(t)\left[\begin{array}{c}
x^{*}(t) \\
\lambda(t)
\end{array}\right] \\
{\left[\begin{array}{c}
x^{*}\left(t_{0}\right) \\
\lambda(T)
\end{array}\right] } & =\left[\begin{array}{c}
0 \\
F x^{*}(T)
\end{array}\right]
\end{aligned}
$$

Note that $x^{*} \equiv 0$ and $\lambda \equiv 0$ is a trivial solution for this TPBVP. The times $\left(t_{0}, T\right)$ are conjugate points if this TPBVP has a non-trivial solution.

Lemma 11. Let $t_{0} \in[0, T]$ be given and assume $\exists \epsilon>0$ such that $J(d) \leq-\epsilon\|d\|_{2,[0, T]}^{2} \forall d \in L_{2}[0, T]$. Then $\left(t_{0}, T\right)$ are not conjugate points of the TPBVP.

Proof. For $t_{0}=T$ the boundary conditions immediately imply $x^{*}(T)=\lambda(T)=0$, i.e. $\left(t_{0}, T\right)$ are not conjugate points. Thus assume $t_{0} \in[0, T)$ and let $\left(x^{*}, \lambda\right)$ be any solution to the TPBVP. Define the signal:

$$
\bar{d}(t):=\left\{\begin{array}{cc}
0 & t \leq t_{0} \\
-R^{-1}(t)\left(S(t)^{T} x^{*}(t)+B(t)^{T} \lambda(t)\right) & t>t_{0}
\end{array}\right.
$$

The TPBVP dynamics can be re-written in terms of $\bar{d}$ :

$$
\left[\begin{array}{c}
\dot{x}^{*}(t) \\
\dot{\lambda}(t)
\end{array}\right]=\left[\begin{array}{cc}
A & 0 \\
-Q & -A^{T}
\end{array}\right]\left[\begin{array}{c}
x^{*}(t) \\
\lambda(t)
\end{array}\right]-\left[\begin{array}{c}
-B \\
S
\end{array}\right] \bar{d}(t)
$$

Moreover, the response of the LTV system (Equation 1) with input $\bar{d}$ and initial condition $x(0)=0$ is given by $x(t)=0$ for $t<t_{0}$ and $x(t)=x^{*}(t)$ for $t \geq t_{0}$.

The cost associated with the input $\bar{d}$ is:

$$
\begin{aligned}
J(\bar{d}) & =x^{*}(T)^{T} F x^{*}(T) \\
& +\int_{t_{0}}^{T}\left[\begin{array}{c}
x^{*}(t) \\
\bar{d}(t)
\end{array}\right]^{T}\left[\begin{array}{cc}
Q(t) & S(t) \\
S(t)^{T} & R(t)
\end{array}\right]\left[\begin{array}{c}
x^{*}(t) \\
\bar{d}(t)
\end{array}\right] d t
\end{aligned}
$$

The integrand can be simplified using the definition of $\bar{d}$ and the TPBVP dynamics in Equation 34:

$$
\begin{aligned}
{\left[\begin{array}{c}
x^{*} \\
\bar{d}
\end{array}\right]^{T}\left[\begin{array}{cc}
Q & S \\
S^{T} & R
\end{array}\right]\left[\begin{array}{c}
x^{*} \\
\bar{d}
\end{array}\right] } & =x^{*^{T}}\left(Q x^{*}+S \bar{d}\right)+\bar{d}^{T}\left(S^{T} x^{*}+R \bar{d}\right) \\
& =-x^{*^{T}}\left(\dot{\lambda}+A^{T} \lambda\right)-\left(\dot{x}^{*}-A x^{*}\right)^{T} \lambda \\
& =-\frac{d}{d t}\left(x^{*^{T}} \lambda\right)
\end{aligned}
$$

These simplifications allow the cost to be rewritten as:

$$
J(\bar{d})=x^{*}(T)^{T} F x^{*}(T)-\int_{t_{0}}^{T} \frac{d}{d t}\left(x^{*^{T}}(t) \lambda(t)\right) d t
$$


Integrate the last term and apply the boundary conditions $x^{*}\left(t_{0}\right)=0$ and $\lambda(T)=F x^{*}(T)$ to show $J(\bar{d})=0$. It is assumed that $J(\bar{d}) \leq-\epsilon\|\bar{d}\|_{2,[0, T]}^{2}$ and hence $\bar{d}=0$. Thus the TPBVP dynamics simplify to:

$$
\left[\begin{array}{c}
\dot{x}^{*}(t) \\
\dot{\lambda}(t)
\end{array}\right]=\left[\begin{array}{cc}
A & 0 \\
-Q & -A^{T}
\end{array}\right]\left[\begin{array}{c}
x^{*}(t) \\
\lambda(t)
\end{array}\right]
$$

The boundary condition $x^{*}\left(t_{0}\right)=0$ thus implies $x^{*} \equiv 0$. This further implies that $\dot{\lambda}=-A^{T} \lambda$ with $\lambda(T)=$ $F x^{*}(T)=0$. Hence $\lambda \equiv 0$. Therefore the TPBVP solution is trivial and $\left(t_{0}, T\right)$ are not conjugate points.

\section{A.2 Proof of $(1 \Rightarrow 2)$}

Assume $J(d) \leq-\epsilon\|d\|_{2,[0, T]}^{2} \forall d \in \mathcal{L}_{2}[0, T]$. Let $\Phi(t, T)$ denote the transition matrix associated with the Hamiltonian dynamics (Equation 32) so that for any $t \in[0, T]$ :

$$
\left[\begin{array}{c}
x^{*}(t) \\
\lambda(t)
\end{array}\right]=\Phi(t, T)\left[\begin{array}{c}
x^{*}(T) \\
\lambda(T)
\end{array}\right]
$$

Note that a solution of the TPBVP must also satisfy the boundary conditions in Equation 33. Next define the following matrix function:

$$
\left[\begin{array}{l}
X_{1}(t, T) \\
X_{2}(t, T)
\end{array}\right]:=\Phi(t, T)\left[\begin{array}{l}
I \\
F
\end{array}\right]
$$

Both $X_{1}$ and $X_{2}$ have $n_{x}$ rows compatible with $\left[\begin{array}{c}x^{*}(t) \\ \lambda(t)\end{array}\right]$.

It can be shown that $X_{1}(t, T)$ is nonsingular for all $t \in[0, T]$. In particular, assume there exists a vector $v$ and time $t_{0} \in[0, T]$ such that $X_{1}\left(t_{0}, T\right) v=0$. Set $x^{*}(T)=v$ and $\lambda(T)=F v$. The state transition matrix (Equation 35) gives a solution $\left(x^{*}, \lambda\right)$ for the Hamiltonian dynamics on $[0, T]$. From the definition $X_{1}$ it follows that $x^{*}\left(t_{0}\right)=X_{1}\left(t_{0}, T\right) v=0$. Hence $\left(x^{*}, \lambda\right)$ satisfy the TPBVP boundary conditions at $\left(t_{0}, T\right)$. By Lemma 11, $J(d) \leq-\epsilon\|d\|_{2,[0, T]}^{2}$ implies that $\left(t_{0}, T\right)$ are not conjugate points, i.e. the solution to the TPBVP is trivial. Thus, $v=x^{*}(T)=0$ so that $X_{1}\left(t_{0}, T\right)$ is nonsingular.

Finally, it can be verified that $Y(t):=$ $X_{2}(t, T) X_{1}(t, T)^{-1}$ satisfies the $\operatorname{RDE}$ and $Y(T)=F$. It follows from $\Phi(T, T)=I$ and Equation 36 that $X_{1}(T, T)=I$ and $X_{2}(T, T)=F$. Hence $Y(T)=F$. Next, differentiating $Y(t)$ with respect to time $t$ yields:

$$
\begin{aligned}
\dot{Y} & =\dot{X}_{2} X_{1}^{-1}-X_{2} X_{1}^{-1} \dot{X}_{1} X_{1}^{-1} \\
& =\left[\begin{array}{ll}
-Y & I
\end{array}\right]\left[\begin{array}{c}
\dot{X}_{1} \\
\dot{X}_{2}
\end{array}\right] X_{1}^{-1}
\end{aligned}
$$

By the definition of $X_{1}$ and $X_{2}$ in Equation 36,

$$
\left[\begin{array}{c}
\dot{X}_{1} \\
\dot{X}_{2}
\end{array}\right]=\dot{\Phi}\left[\begin{array}{l}
I \\
F
\end{array}\right]=H \Phi\left[\begin{array}{l}
I \\
F
\end{array}\right]=H\left[\begin{array}{l}
X_{1} \\
X_{2}
\end{array}\right]
$$

The second equality follows because $\Phi$ is the state transition matrix for $H$. The third equality follows from the definition of $X_{1}$ and $X_{2}$. Combine Equations 37 and 38:

$$
\dot{Y}=\left[\begin{array}{ll}
-Y & I
\end{array}\right] H\left[\begin{array}{c}
I \\
Y
\end{array}\right]
$$

Substitute for $H$ (Eq. 31) to verify $Y$ solves the RDE.

\section{A.3 Proof of $(2 \Rightarrow 1)$}

Assume the RDE has a solution $Y$. The boundary conditions $Y(T)=F$ and $x(0)=0$ imply that $J$ can be equivalently written as:

$$
\begin{aligned}
J(d) & =\int_{0}^{T}\left[\begin{array}{l}
x(t) \\
d(t)
\end{array}\right]^{T}\left[\begin{array}{rr}
Q(t) & S(t) \\
S(t)^{T} & R(t)
\end{array}\right]\left[\begin{array}{l}
x(t) \\
d(t)
\end{array}\right] d t \\
& +\int_{0}^{T} \frac{d}{d t}\left(x(t)^{T} Y(t) x(t)\right) d t
\end{aligned}
$$

The integrand in the second term can be expanded as $\dot{x}^{T} Y x+x^{T} \dot{Y} x+x^{T} Y \dot{x}$. Substitute for $\dot{x}$ using the system dynamics (Equation 1) and for $\dot{Y}$ using the RDE. After some algebra, this yields the following simplified form:

$$
J(d)=\int_{0}^{T}(d(t)-\bar{d}(t))^{T} R(t)(d(t)-\bar{d}(t)) d t
$$

where $\bar{d}:=-R^{-1}(Y B+S)^{T} x$. Thus the cost function can be bounded as follows:

$$
J(d) \leq \alpha\|d-\bar{d}\|_{2,[0, T]}^{2}
$$

where $\alpha:=\max _{t \in[0, T]} \lambda_{\max }(R(t))<0$.

Finally, define the LTV system $W$ with input $d$ and output $d-\bar{d}$ :

$$
W:=\left[\begin{array}{c|c}
A & B \\
\hline R^{-1}(Y B+S)^{T} & I_{n_{d}}
\end{array}\right]
$$

This system is invertible since the feedthrough matrix $I_{n_{d}}$ is nonsingular. Hence $W^{-1}$ exists and has finite gain, i.e. $\|d\|_{2,[0, T]} \leq \beta\|d-\bar{d}\|_{2,[0, T]}$ for some $\beta<\infty$. This further yields $\alpha\|d-\bar{d}\|_{2,[0, T]}^{2} \leq-\epsilon\|d\|_{2,[0, T]}^{2}$ where $\epsilon:=-\frac{\alpha}{\beta^{2}}>0$. Combine this bound with Equation 41 to conclude that $J(d) \leq-\epsilon\|d\|_{2,[0, T]}^{2} \forall d \in \mathcal{L}_{2}[0, T]$

\section{A.4 Proof of $(1 \Rightarrow 3)$}

Assume $J(d) \leq-\epsilon\|d\|_{2,[0, T]}^{2} \forall d \in \mathcal{L}_{2}[0, T]$ with $J$ defined by $(Q, S, R, F)$. As noted previously, the LTV system (1) has finite gain from $d$ to $x$, i.e. $\|x\|_{2,[0, T]} \leq \beta\|d\|_{2,[0, T]}$ for some $\beta<\infty$. Hence, there exists $\tilde{\epsilon}>0$ such that

$$
J(d) \leq-\tilde{\epsilon}\left(\|x\|_{2,[0, T]}^{2}+\|d\|_{2,[0, T]}^{2}\right) \forall d \in L_{2}[0, T]
$$

Define the perturbed cost function $\tilde{J}(d)$ with $(\tilde{Q}, S, R, F)$ where $\tilde{Q}:=Q+\tilde{\epsilon} I_{n_{x}}$. The bound in Equation 43 is 
equivalent to $\tilde{J}(d) \leq-\tilde{\epsilon}\|d\|_{2,[0, T]}^{2} \forall d \in \mathcal{L}_{2}[0, T]$. By $(1 \Rightarrow$ $2)$, there exists $\tilde{Y}:[0, T] \rightarrow \mathbb{S}^{n}$ such that $\tilde{Y}(T)=F$ and

$$
\dot{\tilde{Y}}+A^{T} \tilde{Y}+\tilde{Y} A+\tilde{Q}-(\tilde{Y} B+S) R^{-1}(\tilde{Y} B+S)^{T}=0
$$

Substitute $\tilde{Q}:=Q+\tilde{\epsilon} I_{n_{x}}$ to obtain:

$\dot{\tilde{Y}}+A^{T} \tilde{Y}+\tilde{Y} A+Q-(\tilde{Y} B+S) R^{-1}(\tilde{Y} B+S)^{T}=-\tilde{\epsilon} I_{n_{x}}$

Thus $\tilde{Y}$ satisfies the boundary condition $\tilde{Y}(T) \succeq F$ and satisfies the strict RDI defined with $(Q, S, R, F)$. 